\title{
Rheology of Silica Sols/Gels Obtained by a Y-shaped Reactor with a Dilute Method
}

\author{
Aya KAIDE and Takashi SAEKI \\ Department of Environmental Science and Engineering, \\ Graduate School of Science and Engineering, \\ Yamaguchi University, \\ 2-16-1, Tokiwadai, Ube-shi, Yamaguchi 755-8611, Japan
}

Keywords: Silica Gel, Sol-Gel Transition, Dilute, Gelling Time, Rheology

Silica sols can be continuously produced by the neutralization of sodium silicate with sulfuric acid using a Y-shaped reactor. The viscosity of the produced silica sols gradually increases, and gels are finally formed through the sol-gel transition. The gelation properties are significantly affected by the preparation conditions. Here we examined the effect of the dilution of silica sols by water in terms of the gelation properties of the silica sols and gels, using both dynamic viscoelasticity measurement and the creep test. Information that is needed to control the gelation properties of silica sols and gels for making silica materials into a product is also presented.

\section{Introduction}

Silica sols are generally produced by the neutralization of sodium silicate and sulfuric acid as shown in Eq. (1), which is an easy and convenient progression for industrial mass production.

$$
\begin{aligned}
& \mathrm{Na}_{2} \mathrm{O} \cdot \mathrm{nSiO}_{2}+\mathrm{H}_{2} \mathrm{SO}_{4} \rightarrow \\
& \mathrm{nSiO}_{2}+\mathrm{Na}_{2} \mathrm{SO}_{4}+\mathrm{H}_{2} \mathrm{O}
\end{aligned}
$$

Thus, the obtained amorphous silica is a safe material for both humans and ecosystems, and promising applications include their use as flocculants, absorbents, soil hardeners, retention aids, and more. Generally, silica sols are produced by adding a sodium silicate solution to a batch vessel with the appropriate amount of sulfuric acid. During production, especially at higher $\mathrm{SiO}_{2}$ concentrations (> 10 wt $\%$ ), rapid and localized gelation occurs, which is an obstacle to producing homogenous sols.
Koga and Kosuga (2006) investigated an alternative production method using a Y-shaped reactor, in which diluted sodium silicate and sulfuric acid collide with each other at the intersection of the reactor to ensure complete gelling and avoid localized gelling. Using this method, we can produce homogeneous silica sols continuously with a concentration of up to $14-16 \mathrm{wt} \%$. It is also known that the solution (sol) $\mathrm{pH}$ strongly affects the gelling time. We reported the effects of heating (Kaide et al., 2011) and the existence of a salt (Kaide and Saeki, 2013), which can accelerate the gelation. However, heating cannot change the gels' physical properties such as breaking stress and instantaneous modulus, while the addition of a salt and $\mathrm{pH}$ control dramatically change the gel properties. Koga and Kosuga (2006) reported that dilution of a silica sol by water is effective to control the property of silica sols. This technique has been applied for a water 
flocculation agent, polysilica iron (PSI) flocculation agent (Ehara et al., 2000; Higashi et al., 2004), which is an alternative to polyaluminum chloride (PAC). Saeki et al. (2011) examined the dilution method for acid silica sols and found that the gelling time was shorter than that of a sol with the same silica concentration. However, relatively little work has been published about the effects of dilution on the physical properties of gels.

In this paper, we focus on the dilution method, that is, how the technique can control the physical properties of gels. We evaluated the rheological properties of silica sols and gels by both dynamic viscoelastic measurement and the creep test in order to probe the structure of gels during aging.

\section{Experimental}

Silica sols were produced from sodium silicate (the molecular ratio of $\mathrm{Na}_{2} \mathrm{O}$ to $\mathrm{SiO}_{2}$ was 3.1) with sulfuric acid, where the excess rate of sulfuric acid was set from 1.1 to 1.15. We changed the excess rate of sulfuric acid depending on the concentration of silica. Since a solution of highly loaded silica sometimes shows localized gelling even in the Y-shaped reactor, we increased the excess rate of sulfuric acid to decrease the solution $\mathrm{pH}$. After we obtained a sol from the reactor, we immediately adjusted the concentration of silica and $\mathrm{pH}$, and recorded both.

Figure 1 shows the experimental apparatus for producing silica sols, which consisted of a sulfuric acid line (A) and a sodium silicate line (B). The flow rates of the lines were controlled by inverters installed in each pump (A or B), and the rates could be varied from 11 to $25 \mathrm{~mL} / \mathrm{s}$. Each line was connected to an inlet of a Y-shaped reactor (Figure 2) through a pinhole (diameter $1.2-1.4 \mathrm{~mm}$ ). Since the diameter of the channel at the inlet of the reactor was suddenly reduced by the pinhole, the flow became a jet with an average velocity of 13.2 to $15.2 \mathrm{~m} / \mathrm{s}$. The two jets were forced to collide with each other at the intersection of the reactor. The produced silica sols exited from the outlet of the reactor and went into a beaker. With the Y-shaped reactor, $8 \mathrm{wt} \%$ and $16 \mathrm{wt} \%$ silica sols were prepared. The appropriate amount of water was added to a $16 \mathrm{wt} \%$ sol immediately after the production or at the time when the viscosity of the gel reached $10 \mathrm{mPa} \cdot \mathrm{s}$ (Koga and Kosuga, 2006) to adjust the silica concentration to $8 \mathrm{wt} \%$.

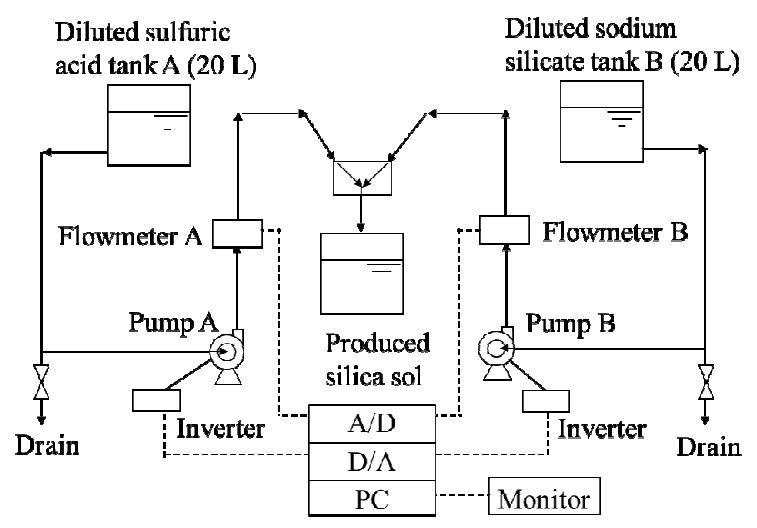

Fig.1 Experimental apparatus

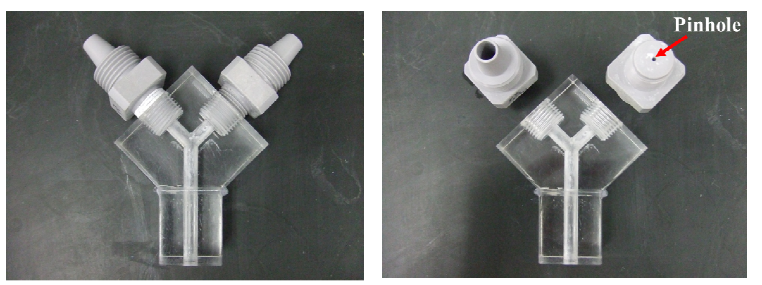

Fig. 2 Y-shaped reactor

The rheological properties of the silica sols were measured using a coaxial-cylinder rotating rheometer (A-300, Elquest Co.). The inner diameter of the outer cup, the diameter of the inner cylinder, and the length of the cylinder were 19.4, 21.0, and $37.4 \mathrm{~mm}$, respectively. The viscoelasticity at the frequency of $1 \mathrm{~Hz}$ and the strain of 0.1 were measured continuously from immediately after manufacture to gelling. 
The physical properties of the gels were measured by using creep test equipment (Rheoner RE2-33005, Yamaden Co.). A cubic gel sample $\left(30 \mathrm{~mm}^{3}\right)$ was pressed by a round plunger with a speed of $0.1 \mathrm{~mm} / \mathrm{s}$. The plunger diameter was $55 \mathrm{~mm}$. The loaded stress of the plunger due to deformation of the sample gel was monitored, and the maximum stress was defined as the breaking stress where the gel broke. A creep test was carried out using a specific load for $60 \mathrm{~s}$, and the load was released. The applied load was set as two-thirds of the breaking stress for each sample. The stress continued to be monitored from the sample load to the recovery, and we obtained the compliance vs. time curve as shown in Figure 3.

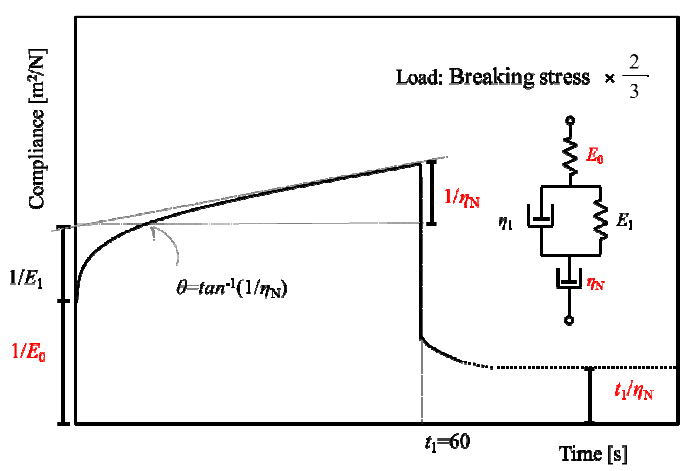

Fig. 3 Creep and recovery curve with four-component model

Here, the compliance was obtained by dividing the strain by the stress, which indicates the deformation rate of a gel sample. We adopted a four-component model as shown in the figure, which consisted of the Maxwell model and the Voigt model, to express the relation between the stress and the time. We focused on both the instantaneous modulus, $E_{0}$ and the Newtonian viscosity, $\eta_{\mathrm{N}}$, which are related for the measured strain. $E_{0}$ is closely related to the early stage of the deformation of the gel, while $\eta_{\mathrm{N}}$ indicates the permanent deformation. Two other parameters, $E_{1}$ and $\eta_{1}$, can demonstrate the curve precisely during the creep and recovery; however, their physical meaning is ambiguous. All of the experiments were conducted at the constant temperature of $25^{\circ} \mathrm{C}$.

\section{Results and Discussion}

The dynamic viscoelastic properties of the $8 \mathrm{wt} \%$ sol during aging are displayed in Figure 4. The axis indicates the storage modulus $G$ ' and the loss modulus $G^{\prime}$, and the abscissa shows the aging time. The point of 0 hour indicates the time when a sol just comes out of the reactor. $G_{-}^{\prime}$ was greater than $G^{\prime}$, and both values gradually increased in the early stage of the gel aging. At the sudden increase in both values, their relation in magnitude reversed, which means that the sample showed elastic rather than viscous properties. The time of the intersection was $41.5 \mathrm{~h}$, defined as the gelling time (Lairez et al., 1992).

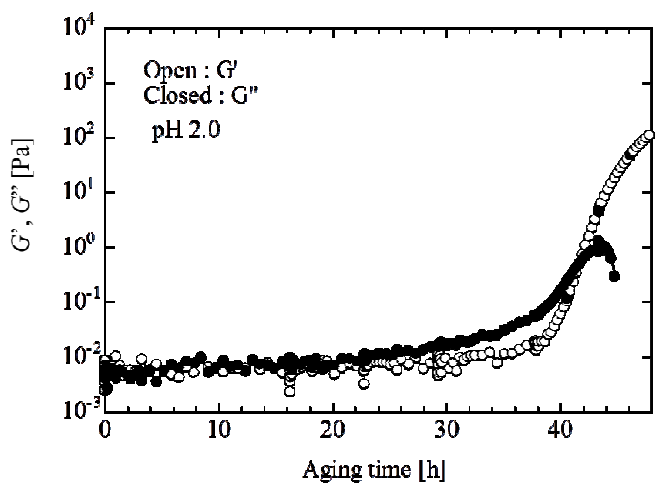

Fig. 4 Dynamic viscoelastic property during gel aging of an $8 \mathrm{wt} \%$ silica sol prepared at $\mathrm{pH} 2.0$

Figure 5 shows the results of dilution experiments evaluated using G' and G'. As the concentration of $\mathrm{SiO}_{2}$ decreased from 16 to $8 \mathrm{wt} \%$, the aging time significantly increased. Since the number of silica particles is large for a dense system, particles easily contact with each other, forming network structure. The 8 wt $\%$ silica sol diluted immediately from $16 \mathrm{wt} \%$ sol (1) showed the 
gelling time of $34.5 \mathrm{~h}$, while that diluted after the apparent viscosity became $10 \mathrm{mPa} \cdot \mathrm{s}(2)$ displayed approx. one-half the gelling time $(15.5 \mathrm{~h})$. It took approx. $4 \mathrm{~h}$ to show the apparent viscosity of $10 \mathrm{mPa} \cdot \mathrm{s}$ for the 16 wt\% sol. In this period, silica particles can easily extend their linkage rather than those in the lower condition. Therefore, the reduction in the gelling time can be explained from the different concentration histories of (1) and (2). We suggest that delaying the dilution timing for high-concentration silica can reduce the gelling time.

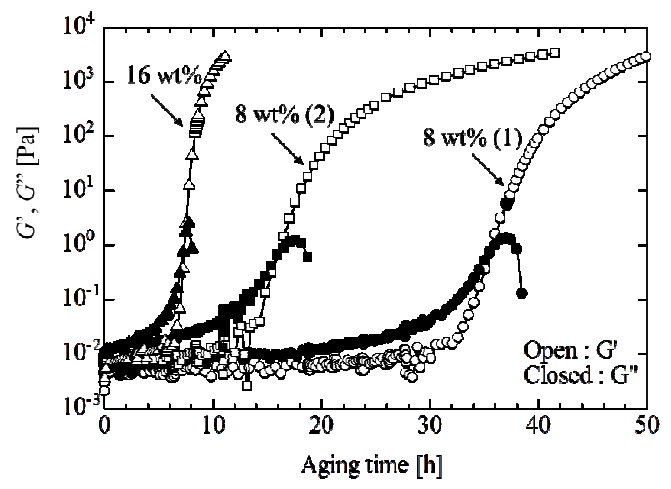

Fig. 5 Effect of dilution on the viscoelasticity of silica sols during gelation:

(1) Diluted just after the production;

(2) Diluted after the apparent viscosity became 10 $\mathrm{mPa} \cdot \mathrm{s}$

Figure 6 shows $E_{0}$ values for the gels prepared with different dilution timing during the aging time, in which $E_{0}$ indicates the degree of deformation of a gel. As for the sol diluted just after silica sol production, $E_{0}$ gradually increased after the gel was formed, which we attribute to the enhancement of the network structure built by the silica particles, and it increased by approx. 10-fold in $24 \mathrm{~h}$. In contrast, the sol diluted when the apparent viscosity became $10 \mathrm{mPa} \cdot \mathrm{s}$ showed a similar trend and strength with the gel diluted just after production.

Figure 7 shows the $\eta_{\mathrm{N}}$ values for the silica gels, which express the permanent deformation in the Maxwell model. Again, a similar trend of $\eta_{\mathrm{N}}$ with respect to the aging time can be seen for the gels obtained with different dilution timings. Thus, dilution timing contributes to the change in the gelation time; however, it does not change the physical properties of the provided gel.

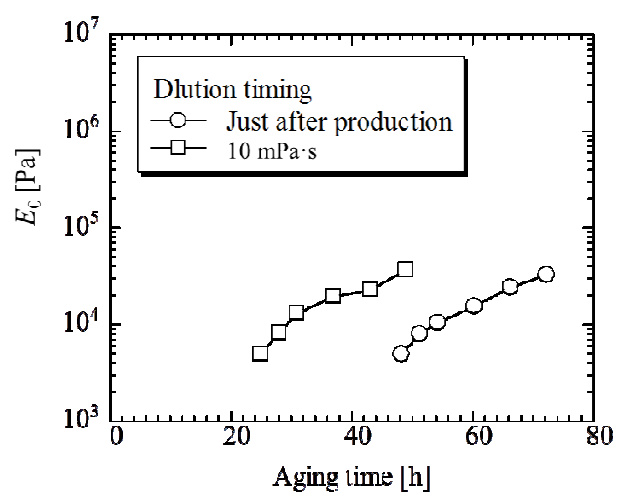

Fig. 6 Results of the dilution experiment evaluated by $E_{0}$

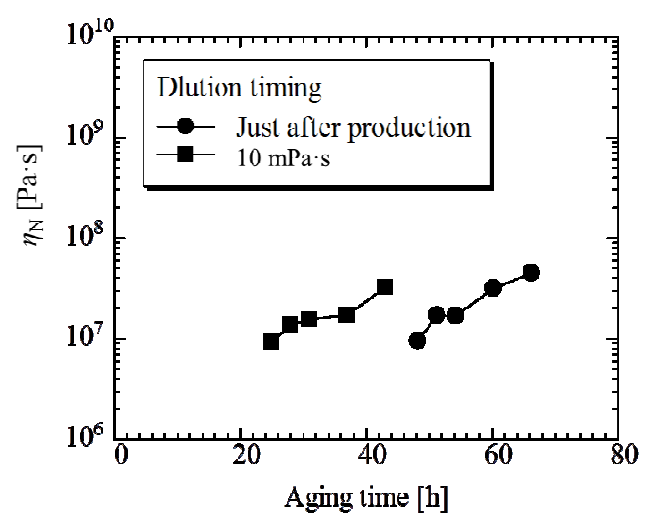

Fig. 7 Results of the dilution experiment evaluated by $\eta_{\mathrm{N}}$

The effect of the dilution method on the gelation properties together with those of other controllable factors revealed in our previous works (Kaide et al., 2011; Kaide and Saeki, 2012; 2013) are summarized in Table 1. Four controllable factors mentioned in the table can change the gelling time; however, both the temperature and the timing of the 
dilution cannot change the gel properties.

The silica sol-gel transition may occur due to the percolation of nanoparticles (Iler, 1979). Munoz-Aguard and Gregorkiewitz (1997) and Knoblich et al. (2001) researched the mechanism of silica sol-gel transition by using ${ }^{29} \mathrm{Si}$ NMR spectra and small angle $\mathrm{X}$-ray scattering, respectively. Mukherjee (1980) used infrared spectroscopy to probe silica gels with different production methods. Beganskiene et al. (2004), Shokri et al. (2013), and Wang et al. (2010) measured the surface properties of silica compounds by Fourier transfer infrared spectroscopy (FTIR). In the present study, we measured the relative ratio of silanol group to siloxane group by means of FTIR (FTIR-8400S, Shimadzu Co., Kyoto, Japan) to focus on the functionalities of silica particles. Sample silica particles were obtained at the onset region of the gelation and after the gelling.

Table 1 Controllable factors of gelation property

\begin{tabular}{c|c|c}
\hline Controllable factor & Gelling time & Gel property \\
\hline Temperature & $\bigcirc$ & $\times$ \\
\hline Timing of dilution & $\bigcirc$ & $\times$ \\
\hline $\mathrm{pH}$ & $\bigcirc$ & $\bigcirc$ \\
\hline Salt $(\mathrm{NaCl})$ & $\bigcirc$ & $\bigcirc$ \\
\hline
\end{tabular}

Figure 8 displays the FTIR spectra of the sample surface. The peaks at 3747,3660 , 3450 and $970 \mathrm{~cm}^{-1}$ indicate the presence of silanol group, which can be interpreted as a non-combination state. The typical siloxane group around 1080, 814 and $459 \mathrm{~cm}^{-1}$ associated with the formation of a condensed silica bounding are also present. The rates of silanol group to siloxane group before and after the gelation were 0.5 (at $40.5 \mathrm{~h}$ ) and 0.375 (at $46.5 \mathrm{~h}$ ), respectively. We found that the siloxane combination increased with the progress of the gelation.

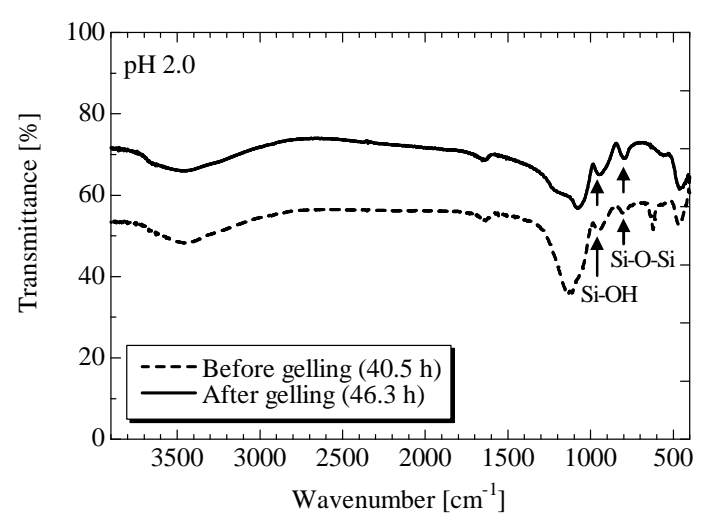

Fig. 8 FTIR spectra of silica particles obtained before and after the gelation

The dilution method examined in this study might not change the surface characteristics of silica particles, and consequently it showed no effect on the gel property at all. Controlling the temperature may also not change the surface nature; however, the gelling reaction is accelerated due to the active Brownian motion at higher temperatures and concentrations, which might increase the collision probability between particles.

\section{Conclusions}

Sol-gel transitions of acid silica sols produced by a Y-shaped reactor were observed in terms of their rheology. We focused on the dilution method to control the silica gelation. The results indicate that the dilution timing contributes to the change in the gelation time, but it does not change the physical properties of the provided gel. We also summarized four factors which can be treated during sol preparation, and we described how these factors can control gelling time and gel property.

\section{Literature Cited}

Beganskiene, A., V. Sirutkaitis, M. 
Kurtinaitiene, R. Juskenas, and A. Kareiva; "FTIR, TEM and NMR Investigations of Stober Silica Nanoparticles," Materials Science, 10, 287-290 (2004)

Ehara, Y., T. Hasegawa, K. Wang, and K. Hashimoto; "Basic Property of Fe-Si inorganic Poly-Silica Flocculation Agent and Its Ability for Water Treatment," Eiseikougaku Sympo ronbunshu, 8, 225-230 (2000) (in Japanese)

Higashi Y., S. Kameda, Y. Ehara, and T. Hasegawa; "Practical Water Treatment by Using Polysilica Iron Flocculation Agent (PSI)," Eiseikougaku Sympo ronbunshu, 12, 129-132 (2004) (in Japanese)

Iler, K. R.; "The Chemistry of Silica," Solubility, Polymerization, Colloid and Surface Properties and Biochemistry of Silica, 172-176, John Wiley and Sons, New York, USA (1979)

Kaide, A., T. Saeki, and S. Kikuchi; "The Effect of Controlling the Aging Condition on the Gelling Property of Silica Sols," Nihon Reoroji Gakkaishi, 39, 205-210 (2011)

Kaide, A. and T. Saeki; "Rheology of Silica Gel Obtained from Alkaline Sol Transition," Nihon Reoroji Gakkaishi, 40, 165-170 (2012)

Kaide, A. and T. Saeki; "Development of a Technique to Control Sol-Gel Transition Using Both Rheological and Morphological Measurements," Advanced Powder Technol. (2013) in printing

Knoblich, B. and Th. Gerber; "Aggregation in $\mathrm{SiO}_{2}$ Sols from Sodium Silicate Solutions," J. Non-Cryst. Solids, 283, 109-113 (2001)

Koga, Y. and M. Kosuga; "New Production for Nano Silica Sol and Its Application for Paper Making," Proc. TAPPI Int. Conf. Nanotechnol., 17-35, Atlanta,
USA (2006)

Lairez, D., M. Adam, J. R. Emery, and D. Durand; "Rheological Behavior of an Epoxy/Amine System near the Gel Point," Macromolecules, 25, 286-289 (1992)

Mukherjee, S. P.; "Sol-Gel Processes in Glass Science and Technology," J. Non-Cryst. Solids, 42, 477-488 (1980)

Munoz-Aguard, M.-J. and M. Gregorkiewitz; "Sol-Gel Synthesis of Microporus Amorphous Silica from Purely Inorganic Precursors," J. Colloid Interface Sci., 185, 459-465 (1997)

Saeki, T., S. Kikuchi, M. Ishida, and A. Kaide; "Production of Acid Silica Sols and Gels by Using a Y-shaped Reactor and Dilution Technique," Int. J. Chem. React. Eng., 9, A22 (2011)

Shokri, B., M. A. Firouzjah, and S. I. Hosseini; "FTIR Analysis of Silicon Dioxide Thin Film Desposited by Metal Organic-Based PECVD," Proc. Int. Plasma Chem. Society, 791 (2013)

Wang, X., P. Wang, Z. Dong, Z. Dong, Z. Ma, J. Jiang, R. Li, and J. Ma; "Highly Sensitive Fluorescence Probe Based on Functional SBA-15 for Selective Detection of $\mathrm{Hg}^{2+}$," Nanoscale Res lett, 5, 1468-1473 (2010) 